\title{
Influence of background characteristics on the optimal sample filling height in gamma-ray spectrometry
}

\author{
H. Ramebäck ${ }^{1}$ iD $\cdot$ T. Vidmar ${ }^{2}$
}

Received: 17 April 2020 / Published online: 12 August 2020

(c) The Author(s) 2020

\begin{abstract}
The minimum detectable activity (MDA) for gamma spectrometric measurements was studied as a function of filling height for two detectors having different background characteristics and for two sample matrices: a water sample with a low content of natural radionuclides, and zircon sand, with high activities of uranium and thorium. The zircon sand is also a material in which the self-attenuation of low-energy gamma photons will be high. The results show that for the water sample it is always favourable to completely fill the sample container. However, due to the inherent content of naturally occurring radionuclides in the zircon sand the MDA may not necessarily improve with the filling height, since an increased amount of sample will increase the background in the spectrum as well.
\end{abstract}

Keywords Gamma spectrometry $\cdot$ Detection limit $\cdot$ Minimum detectable activity $\cdot$ MDA $\cdot$ Optimisation $\cdot$ Filling height

\section{Introduction}

Measurements of radionuclides in environmental and nuclear material are important from both safety and security point of views. One of the most common measurement technique serving this purpose is gamma ray spectrometry [1]. An important characteristic of the gamma spectrometric measurement method is minimum detectable activity (MDA) that defines the least amount of activity in the sample one can be confident of measuring. Beside e.g. the measurement efficiency, amount of sample, measurement time and photon emission probability, the MDA [e.g. $(\mathrm{Bq} / \mathrm{kg})]$ in gamma spectrometry depends on the background level at a specific energy.

Optimisation of measurement geometries has been studied earlier [2-4]. Those studies aimed at maximizing the detection efficiency multiplied by the amount of sample. For a fixed cylindrical sample beaker, the volume or mass is a linear function of the filling height. Increasing the filling height will result in a reduction of the measurement

H. Ramebäck

henrik.ramebeck@foi.se

1 Swedish Defence Research Agency (FOI), CBRN Defence and Security, 16490 Stockholm, Sweden

2 SCK-CEN, Belgian Nuclear Research Centre, Boeretang 200, $2400 \mathrm{Mol}$, Belgium efficiency, $\varepsilon$, but since the amount of sample, $a$, increases with filling height, the product of these quantities might still increase. In the end, and depending on the matrix, i.e. the level of self-attenuation, this function $(\varepsilon \cdot a)$ might in particular at low energies eventually become constant, though $\varepsilon \cdot a$ will never decrease for a fixed sample matrix. What was not considered in the studies cited above was that increasing the amount of sample might also increase the background in the measurement when the dominating background component originates from radionuclides present in the sample matrix other than those to be measured, e.g. naturally occurring radionuclides like ${ }^{40} \mathrm{~K}$ and members of the uranium and thorium decay series.

It should be noted that there are two components contributing to the background in a spectrum. First there is the instrumental background that originates from the detector parts and surroundings of the detector. Then there might be, depending on the sample to be measured, a background component originating form e.g. scattered photons from radionuclides present in the sample. Both these background components will sum up to yield a total background in a spectrum. In this work we have not treated these background components separately, but rather treated them as a total summed background.

For clean samples having in principle no inherent radionuclides present, the background at a specific energy will be fairly constant, i.e. independent of the amount of sample, 
since in this case the background originates from the surroundings of the detector. In those cases the MDA will never deteriorate at any energy with an increased amount of sample. But when the detection limit (in counts) increases due to a higher background more than $\varepsilon \cdot a$ (or when $\varepsilon \cdot a$ ultimately at a low energy becomes constant) the MDA might instead increase with filling height.

In this work MDA was studied as a function of filling height and energy for two different sample matrices, water and zircon sand. The study also included two different detectors having different background characteristics.

\section{Material and methods}

\section{Measurements}

Of the two HPGe (High Purity Germanium) detectors studied one was from Canberra (detector A, Mirion Technologies, Meridien CT, USA) and one from Ortec (detector B, AMETEK, TN, USA). Both detectors had a relative efficiency at $1332 \mathrm{keV}$ of about $80 \%$ and a resolution of about $1.9 \mathrm{keV}$. Detector A has a diameter of $75 \mathrm{~mm}$, and detector $\mathrm{B}$ a diameter of $73.4 \mathrm{~mm}$. The first detector (detector A) was an ultra-low background system consisting of a Canberra 777-B lead shield which was continuously purged with $\mathrm{N}_{2}(\mathrm{~g})$ in order to reduce the background from radon daughters, while detector B had a more ordinary shielding with lead bricks and a copper lining of about $2 \mathrm{~mm}$ in thickness, and no $\mathrm{N}_{2}(\mathrm{~g})$ purging. All electronics of both detectors (high voltage bias supplies, linear amplifiers and multi channel analysers) consisted of analogue NIM modules from Ortec. Measurements of backgrounds as described below were done with the sample container (Noax 200, Sarstedt, Sweden; diameter of $69.5 \mathrm{~mm}$, polypropylene) placed directly on the endcap using Gammavision (Ortec, AMETEK, TN, USA). The detectors were calibrated with a semi-empirical calibration approach described in [5]. No analyte emitting gamma photons in cascade and therefore resulting in count losses was considered in this work. In such cases the MDA will increase accordingly.

Backgrounds were measured for different filling heights (10-60 mm with $10 \mathrm{~mm}$ increments) of blanks, slightly acified MilliQ water samples and zircon sand samples (MinChem HMP Ltd, Stoke-on-Trent, UK). The integral number of counts in the background measurements were evaluated using region of interests (ROI) at different energies ranging from 45 to $1800 \mathrm{keV}$ for detector A. For detector B, the lowest energy considered was $60 \mathrm{keV}$ since the calibration of this detector was only done down to this energy. All measurements were done with a measurement live time of 80 ks (about 22 h).
Based on the semi-empirical calibration [5], efficiencies were calculated for the different filling heights of the sample container using EFFTRAN [6]. The material file in EFFTRAN for the zircon sand was based on the technical information sheet (not a certificate), which stated 99\% $\mathrm{ZrSiO}_{4}+\mathrm{HfSiO}_{4}$. The density of the zircon sand was $2.7 \mathrm{~g} /$ $\mathrm{cm}^{3}$. The chemical content was verified using secondary scanning microscopy with energy dispersive X-ray emission spectroscopy (SEM/EDX, Phenom ProX, ThermoFisher, Germany) and with X-ray diffraction (Bruker D2 Phaser, Bruker, Germany). Detected elements in the EDX analysis was zirconium, silicon and oxygen. From the XRD measurements, the diffractogram was consistent with a reference diffractogram for $\mathrm{Hf}_{0.01} \mathrm{Zr}_{0.99} \mathrm{SiO}_{4}$. In the efficiency transfer (ET), the zircon sand was defined as $100 \% \mathrm{ZrSiO}_{4}$. In this work, 1 at\% of hafnium relative to zirconium had no significant impact on the calculated efficiencies. The zircon sand, measured as received, had according to the SEM measurements particle sizes ranging from about 50-200 $\mu \mathrm{m}$. The aqueous solution was defined as $100 \% \mathrm{H}_{2} \mathrm{O}$ having a density of $1.0 \mathrm{~g} / \mathrm{cm}^{3}$.

\section{Calculations}

The MDA at a given gamma ray energy was calculated according to

$\mathrm{MDA}=\frac{L_{D}}{\varepsilon \cdot a \cdot t_{m} \cdot I_{\gamma}}$

where $\varepsilon$ is the peak efficiency at that energy, $a$ is the amount of the sample (mass or volume) and $t_{\mathrm{m}}$ the measurement time. The photon emission probability $I_{\gamma}$ was always set to unity, i.e. the calculated MDA is valid for a fictitious radionuclide having an $I_{\gamma}$ of unity. $L_{\mathrm{D}}$ is the detection limit in number of counts above the background based on the gross number of counts in the background at the given gamma ray energy (or very close to the energy if background peaks were present), and calculated according to Currie (paired observation) [7].

$L_{D}=2.71+3.29 \cdot \sqrt{2 \cdot N_{B G}}$,

where $N_{\mathrm{BG}}$ is the gross number of counts in the background in an ROI centred on the gamma ray energy. Here it is important to observe the difference between the detection limit $L_{\mathrm{D}}$ expressed as the number of counts above the background, and the MDA. If $L_{\mathrm{D}}$ increases due to a larger sample amount, MDA may necessarily not. Eq. (2) is valid for a risk of false positives and false negatives of 5\%. Uncertainties other than those stemming directly from the measured spectrum may, if large enough, increase the MDA [8, 9]. Such uncertainties can come from e.g. different corrections, but were not considered in this work. 
A short note on the uncertainty in the MDA should be made. According to [10], since the uncertainties on $a$ and $t_{m}$ in Eq. (1) are usually very small and the values used for $\varepsilon$ and $I_{\gamma}$ do not vary between the different measurements of the same sample, the variability of the MDA would be entirely due to the counting uncertainty on the background continuum, resulting in the relative uncertainty of the MDA being $1 / \sqrt{4 \cdot N_{\mathrm{BG}}}$, if we assume that $2.71 \ll 3.29 \cdot \sqrt{2 \cdot N_{\mathrm{BG}}}$, which is also small. Accordingly, and in line with the recommendation from [10], the MDA values are given in this article with no uncertainty.

\section{Results and discussion}

One approach to the optimization of the sample filling height of a given sample container is to look for a maximum in the signal, i.e. the count rate, for a fixed activity content per sample unit (mass, volume) [2, 3]. For a radionuclide that emits gamma photons at an energy $E_{\gamma}$ this can be achieved by maximizing the product of the efficiency and the amount of sample $(\varepsilon \cdot a)$. Using only this criterium, the count rate will never decrease, although the last incremental addition of sample to the sample container might not contribute to the signal. In such a case, one has reached the so called infinite thickness [11] for the sample under measurement. The infinite thickness depends on the sample matrix and the gamma ray energy and is smaller for lower gamma ray energies. However, if the radionuclide to be measured is embedded in a sample matrix that contains other radionuclides and if these emit gamma photons with higher energies than the one to be measured, the background will increase with the amount of sample. By increasing the sample amount one then also increases the background at the energy of interest, since the high energy photon emitters will increase the Compton continuum at lower energies. This effect can be particularly pronounced when the major contribution to the background originates from the sample itself and not from the instrumental background, i.e. the background with no sample presented to the measurement system. Therefore there are two competing processes present in the measurement process with respect to the MDA:

1 the product $\varepsilon \cdot a$ in the denominator in Eq. (1) that never will decrease with sample amount, although ultimately it might become constant when the filling height become large enough, and.

2 the increase of the background with an increased $a$ since more activity other than that of the analyte to be measured is present, which will increase the $L_{\mathrm{D}}$ in the nominator in Eq. (1).
The key question is which of these two quantities will change the most, relatively speaking, when $a$ is increased? If $\varepsilon \cdot a$ increases, on a relative basis, more than $L_{\mathrm{D}}$, the MDA will decrease with $a$, but the contrary applies if $L_{\mathrm{D}}$ increases more. The result in the latter case would then be an increased MDA with the amount of sample.

Figure 1 shows the MDA of detector A for a MilliQ water sample as a function of filling height and for the lowest and the highest gamma ray energy considered in this study.

The same trends were observed for gamma energies between 45 and $1800 \mathrm{keV}$, as well as for detector B having a higher instrumental background. For this sample type it would then always be favourable to measure as much sample as possible.

Figure 2 shows the result from the measurements of the zircon sand on detector A. It can be observed in Fig. 2 that at the lower gamma ray energies $(45,60$ and $120 \mathrm{keV})$ the MDA increases with filling height for the zircon sand. This is a consequence of an increased background at these energies for this material when the sample amount is increased, in combination with a constant or less quickly increasing $\varepsilon \cdot a$. Interestingly, one can actually observe a true minimum in the MDA at $250 \mathrm{keV}$, even though it is not much pronounced. Again, the same results were observed for detector B, which has a higher instrumental background due to a poorer lead shield and no $\mathrm{N}_{2}(\mathrm{~g})$ flowing through the shield. As an illustration, Fig. 3 shows $L_{\mathrm{D}}$ and $\varepsilon \cdot a$ for the zircon sand sample as a function of filling height at 60 and $1800 \mathrm{keV}$ (detector A).

Clearly, at $60 \mathrm{keV} L_{\mathrm{D}}$ increases with filling height while $\varepsilon \cdot a$ remains in principle constant, resulting in an increased MDA, but at $1800 \mathrm{keV}$ both the $L_{\mathrm{D}}$ and the $\varepsilon \cdot a$ increase. In fact, $\varepsilon \cdot a$ increases more than $L_{\mathrm{D}}$ on a relative basis at $1800 \mathrm{keV}$ resulting in a decreased MDA with the filling height.

For a laboratory striving for low minimum detectable activities the choice of the amount of sample depends on the sample matrix and the gamma ray energy emitted by the radionuclide to be measured. Figure 2 shows that it would be

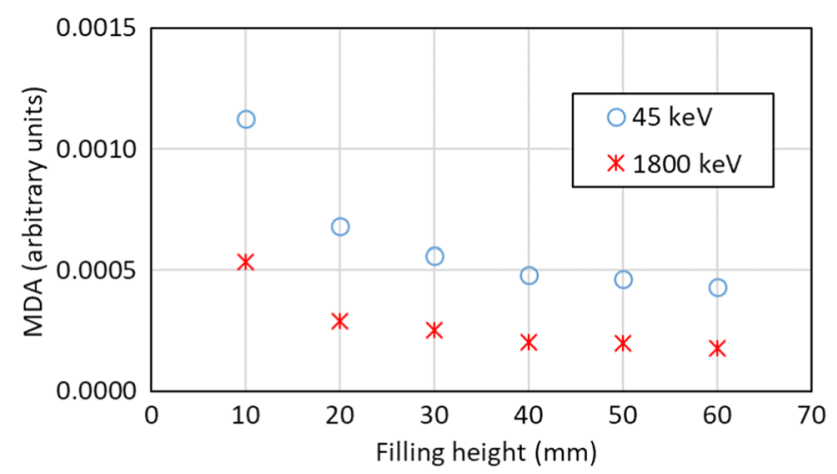

Fig. $1 \mathrm{MDA}$ at 45 and $1800 \mathrm{keV}$ as a function of filling height for a water sample measured on detector A 

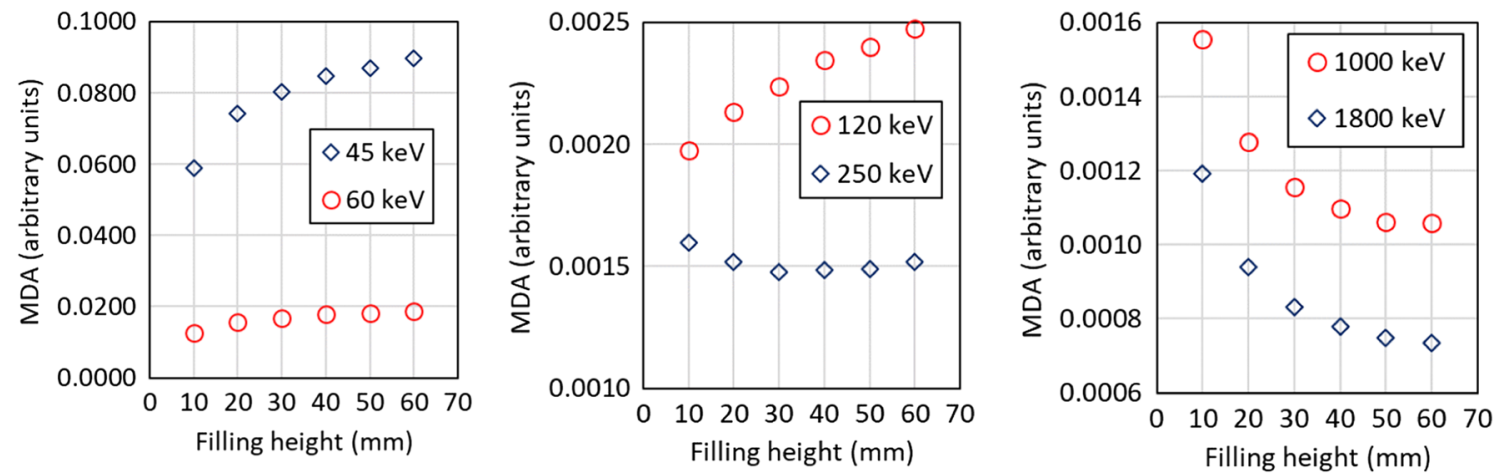

Fig. 2 MDA at 45, 60, 120, 250, 1000 and $1800 \mathrm{keV} \mathrm{keV}$ as a function of filling height for the zircon sand measured on detector A
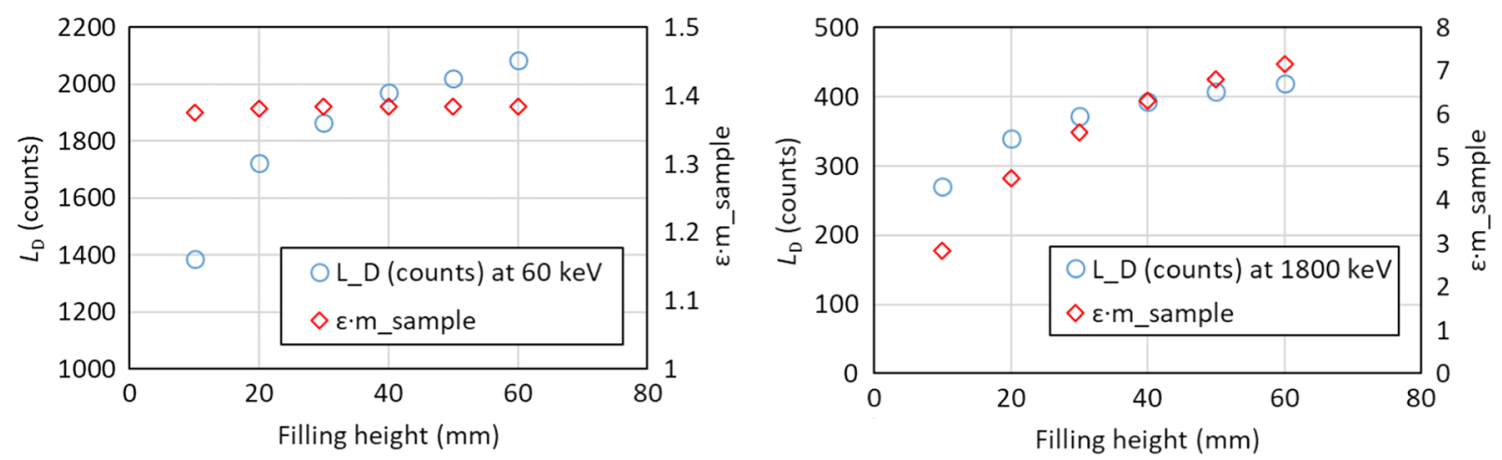

Fig. 3 Detection limit $\mathrm{L}_{\mathrm{D}}$ and $\varepsilon \cdot \mathrm{a}$ (here $\mathrm{a}=\mathrm{m}_{\text {sample }}$ ) as a function of filling height at $60 \mathrm{keV}$ (left figure) and at $1800 \mathrm{keV}$ (right figure) for a zircon sand sample (detector A)

counterproductive to fill the sample beaker to its maximum if a radionuclide emitting a gamma ray with a low energy is to be measured. To a first approximation the MDA would not increase at any energy and for any sample matrix with the amount of sample, but that truly applies only if the background contribution from the sample itself is insignificant. One solution to reach a lower MDA for a low energy gamma emitter would be to change the sample container to one with a larger diameter. However, many laboratories may use only a few container types, since that will reduce the effort and therefore the cost required for calibration.

For many sample types measured in environmental monitoring, radiological preparedness and nuclear forensics it may be favourable from a MDA point-of-view to maximize the sample amount in the measurement even for low-energy gamma emitters. However, this work has shown that such an approach does not always hold if the lowest MDA is desirable. Work done by others that focused on maximizing the count rate did not consider the increased contribution from e.g. natural content of radionuclides in a sample matrix that also has a large self-attenuation [2, 3].

Somewhat hypothetically, in a case where one has the option of choosing between two gamma ray energies pertaining to the same radionuclide, and the two gamma rays have a similar photon emission probability, the adoption of the gamma-ray yielding the lowest MDA differs for the two matrix types studied in this work. For the water matrix the lowest MDA occurs for the energy with the highest peak efficiency, which was at about $120 \mathrm{keV}$, since thereafter the MDA increases with energy in general for all filling heights. However, for all filling heights in the measurements of the zircon sand the results is different. Although the highest peak efficiency occurs at $200 \mathrm{keV}$ in this material, the MDA decreases with energy up to about $500 \mathrm{keV}$ whereafter it is somewhat constant (up to the highest energy of $1800 \mathrm{keV}$ where it is lowest). This can be explained by the rapid increase in the background of the continuum for energies below $500 \mathrm{keV}$ for the zircon sand sample. This MDA energy dependence for detector $\mathrm{A}$ is shown in Fig. 4 for a filling height of $30 \mathrm{~mm}$.

A laboratory involved in free release measurements of radionuclides, radiological preparedness, or nuclear forensics should be aware that they can encounter sample types in combination with low-energy gamma emitters for which it might be counterproductive to measure as much sample as possible. Such awareness might improve (1) the sample 


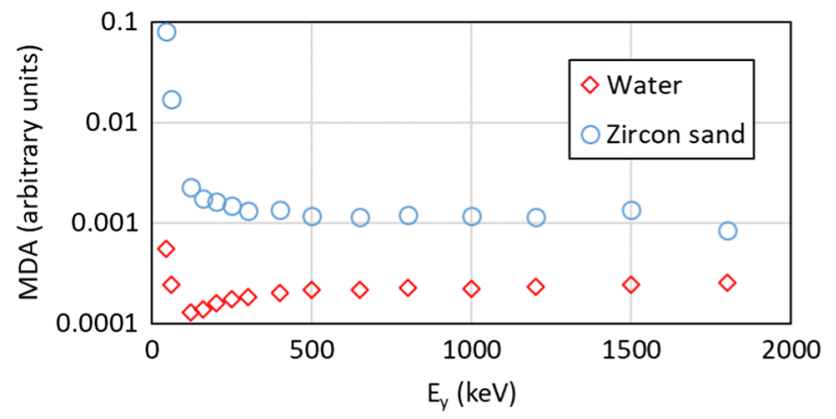

Fig. 4 MDA for the two matrices as a function of gamma ray energy for a filling height of $30 \mathrm{~mm}$. Circles refer to the zircon sand sample and diamonds to the water sample (detector A)

throughput in e.g. free release measurements and radiological preparedness since for a fixed MDA the measurement time can be shortened, and/or (2) in the nuclear security case, the capability to measure lower activity contents of radionuclides that should not be present in a material which is composed of a matrix containing e.g. natural radioactivity.

\section{Conclusions}

In this work it was shown that optimisation of gamma spectrometric measurements via maximizing the count rate for a fixed activity concentration and a fixed cylindrical sample container may not necessarily always improve the MDA. For gamma spectrometric measurements where the background originates from the sample itself, a larger amount of sample may not only increase the $\varepsilon \cdot a$, but also the background and consequently the $L_{\mathrm{D}}$, which then may lead to an increased MDA instead of a decreased one. It was also shown that for some materials there may actually exist, although weak, a true optimum in the filling height with respect to MDA, depending on the gamma ray energy to be measured. Moreover, there is a dilemma present when the analyst wants to measure radionuclides emitting low energy gamma photons in combination with radionuclides emitting high energy gamma photons. In such cases the MDA may decrease with the amount of sample for the high energy emitter, but increase for the low energy one.

Acknowledgements Open access funding provided by Swedish Defence Research Agency. This work was funded by the Swedish Ministry of Defence, Project No. A405720.

Open Access This article is licensed under a Creative Commons Attribution 4.0 International License, which permits use, sharing, adaptation, distribution and reproduction in any medium or format, as long as you give appropriate credit to the original author(s) and the source, provide a link to the Creative Commons licence, and indicate if changes were made. The images or other third party material in this article are included in the article's Creative Commons licence, unless indicated otherwise in a credit line to the material. If material is not included in the article's Creative Commons licence and your intended use is not permitted by statutory regulation or exceeds the permitted use, you will need to obtain permission directly from the copyright holder. To view a copy of this licence, visit http://creativecommons .org/licenses/by/4.0/.

\section{References}

1. Knoll GF (1999) Radiation detection and measurement, 3rd edn. Wiley, New York

2. Klemola S (1996) Optimization of sample geometries in lowlevel gamma spectroscopy. Nucl Instrum Methods Phys Res 369:578-581

3. Barrera M, Ramos-Lerate I, Ligero RA, Casas-Ruiz M (1999) Optimization of sample height in cylindrical geometry for gamma spectrometry measurements. Nucl Instrum Methods Phys Res 421:163-175

4. Ahmed AS, Capello K, Chiang A, Cardenas-Mendez E, Kramer GH (2009) Optimization of geometric parameters for Marinelli beaker to maximize the detection efficiency of an HPGe detector. Nucl Instrum Methods Phys Res 610:718-723

5. Jonsson S, Vidmar T, Ramebäck H (2015) Optimization of geometric parameters for Marinelli beaker to maximize the detection efficiency of an HPGe detector. J Radioanal Nucl Chem 303:1727-1736

6. Vidmar T (2005) EFFTRAN-a Monte Carlo efficiency transfer code for gamma-ray spectrometry. Nucl Instrum Methods 550:603-608

7. Currie LA (1968) Limits for qualitative detection and quantitative determination. Anal Chem 40:586-593

8. ISO 11929-1 (2019) Determination of characteristic limits (decision threshold, detection limit and coverage interval) for measurements of ionizing radiation - fundamentals and applications - part 1: elementary applications. The International Organization for Standardization, Geneva

9. Ramebäck H, Persson L, Ekberg C, Lindgren P, Bruggeman M (2020) Calculation of decision threshold and detection limit in radiometric measurements using a Monte Carlo method. Appl Radiat Isot. https://doi.org/10.1016/j.apradiso.2019.108949

10. Gilmore G (2008) Practical gamma-ray spectrometry, 2nd edn. Wiley, Chichester

11. Sprinkle JK Jr, Christiansen A, Cole R, Collins ML, Hsue S-T, Knepper PL, McKown TO, Sieblelist R (1997) Low-resolution gamma-ray measurements of uranium enrichment. Appl Radiat Isot 48:1525-1528

Publisher's Note Springer Nature remains neutral with regard to jurisdictional claims in published maps and institutional affiliations. 